\title{
Comparación de Áreas Verdes Próximas a Instituciones Educativas en dos distritos de Lima Metropolitana, 2019
}

\author{
Comparison of Green Areas Near Educational Institutions in two districts of \\ Metropolitan Lima, 2019
}

\author{
Marcos Espinoza-Pereyra ${ }^{1}$, Giovanna Gutiérrez-Medina ${ }^{2}$, Nora Malca-Casavilca ${ }^{3}$ \\ Recibido: 01/07/2020 - Aprobado: 30/10/2020 - Publicado: 30/11/2020
}

\begin{abstract}
RESUMEN
La investigación busca establecer una comparación entre la presencia de áreas verdes próximas a centros educativos en dos distritos de Lima Metropolitana, Breña y Pueblo Libre, debido a la influencia que tienen en el bienestar humano; el trabajo incluyó visitas de campo, in situ de ambos distritos y un trabajo de gabinete donde se procesó la información tomada con el GPS Garmin Etrex 20, mediante el programa Postgresql / Postgis, además del uso del programa Google Earth Pro y la descripción de los datos obtenidos en las salidas de campo. Se determinó que Pueblo Libre posee un mayor porcentaje de área total parque (y en consecuencia, una mayor cantidad de área verde), por lo que la relación de parques con la cantidad de entidades educativas a radios de 100, 200 y 300 m en Pueblo Libre es mayor; Breña posee $0.33 \mathrm{~m}^{2}$ de área verde por habitante y Pueblo Libre $2.14 \mathrm{~m}^{2}$.
\end{abstract}

Palabras claves: Áreas verdes urbanas; instituciones educativas; geomarketing, Breña; Pueblo Libre.

\begin{abstract}
The research seeks to establish a comparison between the presence of green areas near educational centers in two districts of Metropolitan Lima, Breña and Pueblo Libre, due to the influence they have on human well-being; the work included field work, with institutional visits to both districts and a cabinet work where the information taken with the Garmin Etrex 20 GPS using Postgresq//Postgis software, besides Google Earth Pro program and the description obtained in the field work was processed. It was determined that Pueblo Libre has a higher percentage of total park area (and consequently, a greater amount of green area), so the relationship of parks with the number of educational entities at radii of 100,200 and $300 \mathrm{~m}$ in Pueblo Free is greater; Breña has $0.33 \mathrm{~m} 2$ of green area per inhabitant and Pueblo Libre $2.14 \mathrm{~m}^{2}$.
\end{abstract}

Keywords: Urban green areas; educational institutions; geomarketing; Breña; Pueblo Libre.

1 Ingeniero Geógrafo de la Universidad Nacional Mayor de San Marcos. Lima, Perú. Magister en Ciencias Ambientales con mención en Gestión y Ordenamiento Ambiental del Territorio. Autor para correspondencia: mfespinozapereyra@gmail.com ORCID: https://orcid.org/0000-0001-9332-5450

2 Geógrafa de la Universidad Nacional Mayor de San Marcos. Lima, Perú. Bachiller egresada de la Maestría en Ciencias Ambientales con Mención en Gestión y Control de la Contaminación. E-mail: blumme200@hotmail.com ORCID: https://orcid.org/0000-0002-4827-0531

3 Docente de la Universidad Nacional Mayor de San Marcos. Lima, Perú. Unidad de Posgrado. Magister en Ciencias Ambientales con mención en Control de la Contaminación y Ordenamiento Territorial. E-mail: noramalcac@gmail.com ORCID: https://orcid.org/0000-0002-2625-9008 


\section{INTRODUCCIÓN}

Es evidente la transformación del ecosistema urbano a lo largo del tiempo, y los factores responsables son la ubicación geográfica del distrito, las condiciones meteorológicas, el crecimiento y desarrollo urbano, los indicadores urbanos de la calidad del espacio público como la calle, los lugares de encuentro y circulación, las áreas verdes y los elementos de valor cultural e histórico, los indicadores socio-económicos, culturales y políticos que a través de la historia ejercen un papel decisivo sobre la expresión física y valores de las áreas verdes urbanas. La rápida urbanización ha alterado la cantidad de áreas verdes, que se ha comprobado tienen influencia en la salud de la población (Nieuwenhuijsen, M.J., Khreis, H, TrigueroMas, M., Gascón, M., Dadvand, 2017). Como lo cita Capristán Flores (2017) la responsabilidad de la gestión de las áreas verdes recae en los gobiernos municipales, el déficit de estas ocasiona riesgos de exclusión social, afecta el valor monetario y de servicios ecosistémicos que brindan, por lo que cada comunidad debe adecuar la gestión de sus espacios verdes teniendo en cuenta las variables sociales, económicas y culturales.

Pérez (2011) afirma que el paisaje urbano de una ciudad es la expresión de la relación de las condiciones de orden social y económico de los habitantes, es decir, su diversidad y estado obedecen en gran medida a la estratificación socio-espacial de la ciudad ya que existe, según este autor, una marcada estratificación socio espacial entre las áreas marginales, las áreas de vivienda popular, las de clase media y las de vivienda suntuaria, expresada en la diferencia de la calidad del espacio público, la estética urbana y estado de las edificaciones, carencia y deterioro de zonas verdes y arborización urbana y deterioro de los elementos del entorno natural. En nuestra realidad también se debe considerar, según lo afirma Meza Ortega (2018) además de los aspectos citados líneas arriba, que para colegios de régimen estatal la aplicación e información, protección e importancia de las áreas verdes es muy escasa dentro del ámbito educativo, además existe una currículo establecido para la Educación Básica Regular (EBR) que puede ser un limitante para proyectos de áreas verdes.

Según Wolch, J. R., Byrne, J., y Newell (2014), las áreas verdes promueven la actividad física, bienestar psicológico y salud de los residentes próximos a estas. A la fecha se puede citar el estudio de Markevych et al. (2014) donde se evidenció una asociación inversa entre el verdor residencial y la presión sanguínea en niños. Xiang Xiao et al. (2020) afirma que el verdor alrededor de la escuela de un niño puede ser importante, debido a que uno de los mecanismos sugeridos para este vínculo es el aumento de la actividad física (Jia et al., 2018), el mantenimiento de un peso corporal saludable (Sander et al., 2017), alivio del estrés y otras actividades recreativas (Herrera et al., 2018); (Van Aart et al., 2018). En Lima Metropolitana, distritos como el Agustino (Ayón, 2017) están desarrollando iniciativas que buscan colocar como ejes transversales la importancia de los espacios naturales y dentro de estos están las áreas verdes, otros distritos han implementado estrategias para incrementar la oferta de áreas verdes, estas incluyen el reverdecimiento de la tierra urbana remanente y el mejoramiento de las áreas verdes existentes (LIMA CÓMO VAMOS, 2014). La creación de nuevos espacios verdes puede hacer que los vecindarios sean más saludables y estéticamente más atractivos, también puede aumentar los costos de vivienda y el valor de las propiedades. Los planificadores urbanos, los diseñadores y los ecologistas, por lo tanto, deben centrarse en las estrategias de áreas verdes urbanas que sean "lo suficientemente verdes", como actualmente ocurre en el norte y sur chico, y que protejan explícitamente la sostenibilidad social y ecológica. El objetivo de la investigación es hacer una comparación de áreas verdes urbanas próximas a las instituciones educativas de Breña y Pueblo Libre como unidad de análisis.

Atendiendo lo mencionado en el párrafo anterior, en la Tabla 1 se muestra datos comparativos de los distritos de Breña y Pueblo Libre como también datos de la provincia de Lima, de los cuales para cada entidad administrativa se tiene el área estimada proveniente del Instituto Nacional de Estadística e Informática, población total y número de hogares estimado al 2019 (Compañía peruana de estudios de mercados y opinión pública S.A.C, 2019), el número de entidades educativas de nivel inicial, primaria y secundaria de acuerdo al Ministerio de Educación del Perú - Estadística de Localidad Educativa (ESCALE, 2019), y el número de parques que fue contabilizado en las salidas de campo.

De acuerdo con la Tabla 1, el número de entidades educativas se contabiliza según el nivel de enseñanza: si en una entidad educativa existen tres niveles (inicial, primaria y secundaria), luego, se contabiliza como si fueran tres (ESCALE, 2019), a su vez, se tiene el número de entidades educativas por nivel (inicial, primaria y secundaria) y gestión (pública o privada) como también el número de estudiantes a nivel de entidad educativa. En la Tabla 2 se tiene un comparativo del número de entidades educativas por nivel entre los distritos de Breña y Pueblo Libre y en la Tabla 3 se tiene un comparativo del número de estudiantes entre los mencionados distritos.

Tabla 1. Datos generales de los distritos de Breña, Pueblo Libre y la provincia de Lima.

\begin{tabular}{lccccc}
\hline Entidad administrativa & Área $^{\mathbf{1}}\left(\mathbf{k m}^{2}\right)$ & $\begin{array}{c}\text { Población total } \\
\text { (miles) }\end{array}$ & $\begin{array}{c}\text { Número de hogares } \\
\text { (miles) }\end{array}$ & $\begin{array}{c}\text { Número de entidades } \\
\text { educativas }^{3}\end{array}$ & Número de parques $^{4}$ \\
\hline Distrito de Breña & 3.21 & 93.4 & 26.2 & 137 & 21 \\
Distrito de Pueblo Libre & 4.64 & 90.7 & 27.0 & 109 & 73 \\
Provincia de Lima & 2697.57 & 9480.5 & 2444.0 & - & - \\
\hline
\end{tabular}

Fuentes: 1. Instituto Nacional de Estadística e Informática; 2. (Compañía peruana de estudios de mercados y opinión pública S.A.C, 2019); 3. (ESCALE, 2019); 4 Elaboración propia. 
Tabla 2. Comparativo de las entidades educativas de los distritos de Breña y Pueblo Libre según el nivel de enseñanza.

\begin{tabular}{lcccccc}
\hline \multirow{2}{*}{ Nivel } & \multicolumn{3}{c}{ Breña } & \multicolumn{3}{c}{ Pueblo Libre } \\
\cline { 2 - 7 } & Publica & Privada & Total & Publica & Privada & Total \\
\hline Inicial & 9 & 45 & 54 & 5 & 51 & 56 \\
Primaria & 10 & 37 & 47 & 7 & 25 & 32 \\
Secundaria & 5 & 31 & 36 & 3 & 18 & 21 \\
Total & 24 & 113 & 137 & 15 & 94 & 109 \\
\hline
\end{tabular}

Fuente. (ESCALE, 2019)

Tabla 3. Comparativo de las entidades educativas de los distritos de Breña y Pueblo Libre según el número de estudiantes.

\begin{tabular}{lcccccc}
\hline \multirow{2}{*}{ Nivel } & \multicolumn{3}{c}{ Breña } & \multicolumn{5}{c}{ Pueblo Libre } \\
\cline { 2 - 7 } & Publica & Privada & Total & Publica & Privada & Total \\
\hline Inicial & 1602 & 2633 & 4235 & 1028 & 2006 & 3034 \\
Primaria & 3302 & 6650 & 9952 & 2795 & 4484 & 7279 \\
Secundaria & 2959 & 6998 & 9957 & 1398 & 3613 & 5011 \\
Total & 7863 & 16281 & 24144 & 5221 & 10103 & 15324 \\
\hline
\end{tabular}

Fuente. (ESCALE, 2019)

\section{MÉTODOS}

La metodología del trabajo comprendió los siguientes pasos:

\subsection{Planificación del trabajo de campo}

La base para este fue la información cartográfica (un mapa) de las entidades educativas del UGEL $03^{4}$ que comprende los distritos de Cercado de Lima, Breña, Jesús María, La Victoria, Lince, Magdalena del Mar, Pueblo Libre, San Isidro y San Miguel siendo publicada por el Ministerio de Educación del Perú - Estadística de Localidad Educativa (ESCALE, 2012). De acuerdo con la información cartográfica mencionada, se preparó dos mapas correspondientes a los distritos de Breña y Pueblo Libre a la escala $1 / 10000$, proyección cartografía UTM zona $18 \mathrm{~S}$ y datum WGS84 (siendo los parámetros cartográficos iguales al mapa publicada por ESCALE (2012).

\subsection{Trabajo de campo}

Según los objetivos de esta investigación (comparación de los parques próximos a las entidades educativas de los distritos de Breña y Pueblo Libre), el trabajo de campo fue visita directa a los parques para verificar que instituciones educativas estaban próximas en un radio de 2 a 3 manzanas (dependiendo de la extensión de la cuadra misma); también depende del tiempo promedio sugerido en ir hacia el parque más próximo que serían 15 minutos (Sorensen et al., 1998); Comisión Nacional de Medio Ambiente (CONAMA, 2002) y citados por (Flores-Xolocotzi \& González-Guillén, 2019). Para el distrito de Breña se realizaron dos días de visita de campo y para Pueblo Libre se realizaron tres días; en cada día se tomó un promedio de 4 horas. Como herramientas de trabajo se tuvieron los dos mapas mencionados en el párrafo anterior (Planificación del trabajo de campo), un GPS Garmin Etrex 20 (para obtener la coordenada del parque y de la entidad educativa), una cámara fotográfica Sony (para el registro fotográfico del parque y de la entidad educativa), un cuaderno y lapicero para los apuntes respectivos.

4 Abreviatura de Unidad de Gestión Educativa Local Numero 3.
Dado que la información cartográfica de ESCALE es del (2012), se observó que algunas instituciones educativas no existían como tales, encontrándose también nuevas o se movieron de local, por lo tanto, con esta investigación se recomienda actualizar la información cartográfica de Breña y Pueblo Libre de las entidades educativas.

En el trabajo de campo se tomó en consideración los siguientes servicios y mencionadas en la Tabla 4, que muestran algunas propiedades visibles de los parques que reflejan o describen su condición actual como un lugar para el recreo y/o descanso para la población.

\subsection{Procesamiento de la información cartográfica}

Para esta etapa se obtuvo lo siguiente:

- Entidades educativas: se procesó la información tomada del GPS Garmin Etrex 20 creando un archivo shapefile de las entidades educativas para el distrito de Breña y Pueblo Libre con atributos tales como Nombre, Dirección, Nivel educativo (jardín, primaria, secundaria y alternativo), Gestión (pública o privada) y Administración (sector educación, particular o parroquial); parte de esta información es tomada del Ministerio de Educación del Perú - Estadística de localidad Educativa (ESCALE, 2019). Para el análisis de distancia se exporto el shapefile de entidades educativas al motor de base de datos Postgresql / Postgis obteniéndose una tabla geoespacial.

- Parques: utilizando el programa Google Earth Pro, se digitalizo los parques de los distritos de Breña y Pueblo Libre y también el área verde de los parques de los mencionados distritos obteniéndose dos archivos shapefile respectivamente y con la descripción obtenida en las salidas de campo. Para el análisis de distancia se exporto el shapefile de los parques al motor de base de datos Postgresql / Postgis obteniéndose dos tablas geoespaciales. 


\section{RESULTADOS}

Como primer resultado se tiene la Tabla 5 que muestra un resumen comparativo, en la primera columna el número de parques existentes en cada distrito, además el área total de parque teniendo el total del área de los parques (entendiéndose como la suma del área verde del parque más las aceras, accesos, entre otros elementos que conforman un parque), el área mínima y máxima de parque para cada distrito y a su vez se tiene el área verde de los parques (el área de césped o pasto) y de igual forma se tiene el área total, el área mínima y máxima estimadas.

En la Tabla 6 se tiene el número de las entidades educativas de ambos distritos asociados con las áreas verdes próximos a estos, que fueron identificadas y registrados en las salidas de campo mostradas por nivel y gestión educativa.
En la Figura 1 se tiene el mapa de las entidades educativas visitadas en las salidas de campo

En la Tabla 7 se tiene en la primera columna el estimado del área verde de los parques por distrito y en la segunda columna la población distrital (Compañía peruana de estudios de mercados y opinión pública S.A.C, 2019). Seguidamente se tiene el estimado de la relación Área / Población, seguidamente lo que muestra el Ministerio del Ambiente del Perú - Sistema Nacional de Información Ambiental (SINIA, 2019) y por último lo que recomienda la Organización Mundial de la Salud (OMS, tomado de Lamela, A., 2011) y a su vez citado por Vera, L., Ramírez, M. y Pértile (2013), ya que es de importancia tener una medida del área verde por persona siendo esta última importante tanto para el embellecimiento paisajístico y fomentar actividades físicas y bienestar psicológico (Wolch, J. R., Byrne, J., y Newell, 2014).

Tabla 4. Servicios, valores, puntaje y descripción para la comparación de los parques de los distritos de Breña y Pueblo Libre

\begin{tabular}{|c|c|c|c|}
\hline Servicios & Valores & Puntaje & Descripción \\
\hline \multirow{3}{*}{ Cuidado de la vegetación } & Sana y cuidada casi al $100 \%$ & 3 & \multirow{3}{*}{ Mantenimiento en la poda del césped y riego. } \\
\hline & Sana y cuidada casi al $50 \%$ & 2 & \\
\hline & Sana y cuidada menor al $50 \%$ & 1 & \\
\hline \multirow{2}{*}{ Acceso } & Abierto al publico & 2 & \multirow{2}{*}{ Tiene rejas o algún impedimento al público en general. } \\
\hline & Cerrado al publico & 1 & \\
\hline \multirow{2}{*}{ Juego para niños } & Con juegos & 2 & \multirow{2}{*}{ Contiene juegos para niños. } \\
\hline & Sin juegos & 1 & \\
\hline \multirow{2}{*}{ Seguridad } & Con seguridad & 2 & \multirow{2}{*}{ Cuenta con una caseta de serenazgo, vigilancia, entre otros. } \\
\hline & Sin seguridad & 1 & \\
\hline \multirow{2}{*}{ Basura } & Con recojo de basura e implementado con tacho & 2 & \multirow{2}{*}{ Presencia de basuras, desperdicios en general } \\
\hline & Sin recojo de basura e implementado con tacho & 1 & \\
\hline \multirow{3}{*}{ Ornato } & Veredas y bancas en buen estado casi al $100 \%$ & 3 & \multirow{3}{*}{$\begin{array}{l}\text { Presencia de veredas, bancas y demás elementos encontrados } \\
\text { en un parque. }\end{array}$} \\
\hline & Veredas y bancas en buen estado casi al $50 \%$ & 2 & \\
\hline & $\begin{array}{l}\text { Veredas y bancas en buen estado menor al } 50 \% \text { o } \\
\text { ausencia de las mismas }\end{array}$ & 1 & \\
\hline
\end{tabular}

Fuente: Elaboración propia.

Tabla 5. Resumen comparativo de las áreas de los parques de los distritos de Breña y Pueblo Libre.

\begin{tabular}{|c|c|c|c|c|c|c|c|}
\hline \multirow{2}{*}{ Distrito } & \multirow{2}{*}{$\begin{array}{l}\text { Numero } \\
\text { parques }\end{array}$} & \multicolumn{3}{|c|}{ Área total parque $\left(\mathrm{m}^{2}\right)$} & \multicolumn{3}{|c|}{ Área verde parque $\left(\mathrm{m}^{2}\right)$} \\
\hline & & Total & Mínimo & Máximo & Total & Mínimo & Máximo \\
\hline Breña & 21 & 47,202 & 337 & 5,618 & 30,567 & 162 & 4,366 \\
\hline Pueblo Libre & 73 & 296,555 & 604 & 26,459 & 194,312 & 250 & 17,982 \\
\hline
\end{tabular}

Fuente: Elaboración propia.

Tabla 6. Comparativo de las entidades educativas visitadas en las salidas de campo de los distritos de Breña y Pueblo Libre según el nivel de enseñanza.

\begin{tabular}{lccccc}
\hline & & Breña & & \multicolumn{2}{c}{ Pueblo Libre } \\
\cline { 2 - 6 } & Publica & Privada & Total & Publica & Privada \\
\hline Inicial & 6 & 29 & 35 & 4 & 47 \\
Primaria & 4 & 16 & 20 & 7 & 31 \\
Secundaria & 4 & 10 & 14 & 32 & 16 \\
Total & 14 & 55 & 69 & 14 & 88 \\
\hline
\end{tabular}

Fuente: Elaboración propia. 


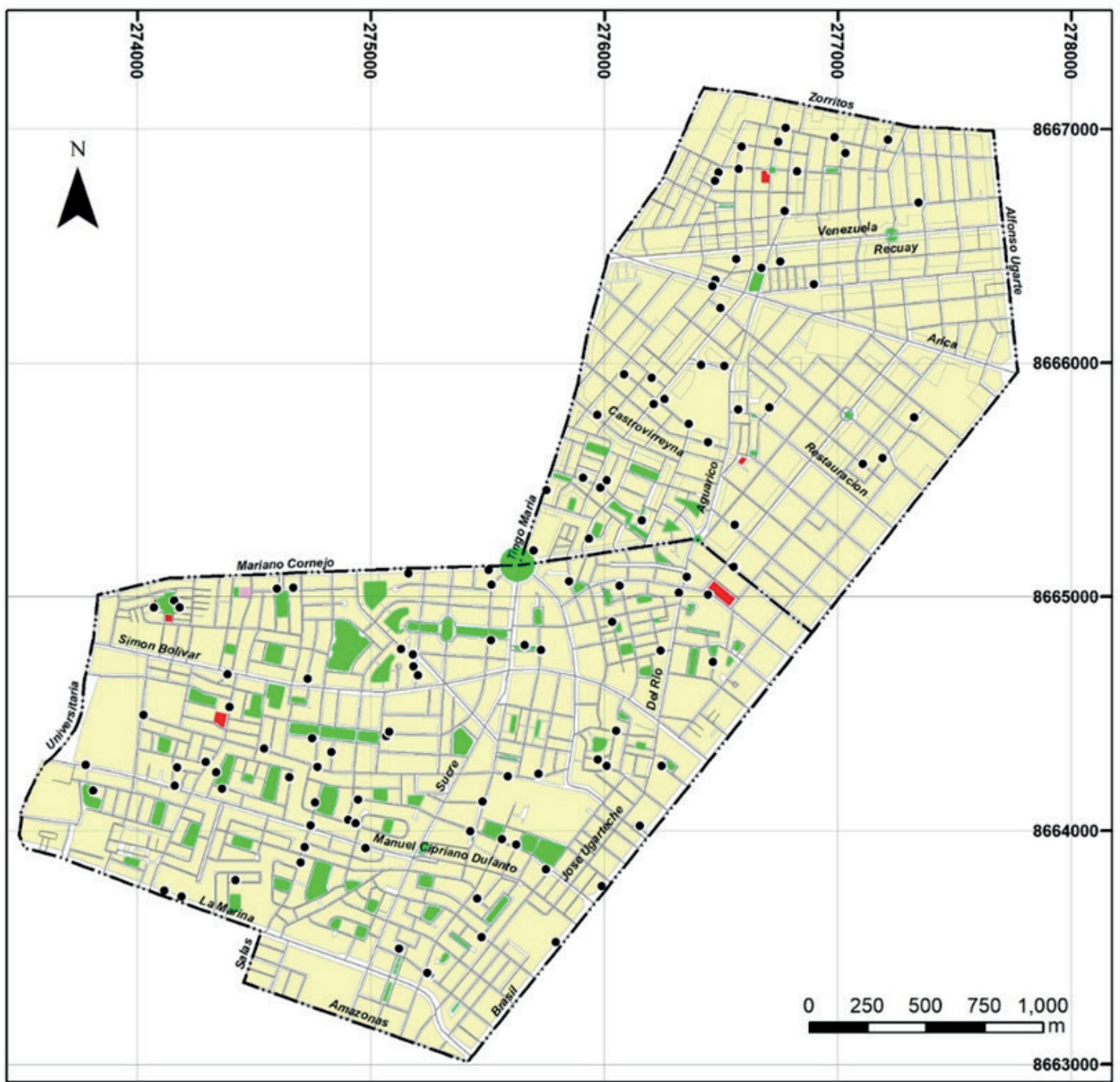

\section{LEYENDA}

- Entidades Educativas visitadas Vias

$-\cdot-$ Límite distrital

\section{Manzanas}

Deporte

Sitio arqueológico

Vivienda / Comercio

Parque
MAPA DISTRITAL DE BREÑA Y PUEBLO LIBRE

UBICACIÓN DE ENTIDADES EDUCATIVAS Y PARQUES

datos cartográficos

datos administrativos

Escala: $1 / 25,000$

Proyección: UTM $18 \mathrm{~S}$ Provincia: Lima

Datum: WGS 84

Distrito: Breña y Pueblo Libre

Fecha: agosto 2019

Figura 1. Mapa de los distritos de Breña y Pueblo Libre con las entidades educativas visitadas.

Fuente: Elaboración propia.

Tabla 7. Comparativo del estimado de la relación "Área verde de los parques / Población" de los distritos de Breña y Pueblo Libre.

\begin{tabular}{lccccc}
\hline \multirow{2}{*}{ Distrito } & \multirow{2}{*}{ Área verde de los parques $\left(\mathrm{m}^{2}\right)$} & Población (en miles) & \multicolumn{3}{c}{ Área / Población $\left(\mathrm{m}^{2} / \mathrm{hab}\right)$} \\
\cline { 4 - 6 } & & & Estimado & SINIA & OMS \\
\hline Breña & 30,567 & 93.4 & 0.33 & 1.01 & 10 \\
Pueblo Libre & 194,312 & 90.7 & 2.14 & 3.74 & 10 \\
\hline
\end{tabular}

Fuente: Elaboración propia. 
El análisis de los servicios en los parques del distrito de Breña y Pueblo Libre se realizó teniendo como base la Tabla 4 (Servicios, valores, puntaje y descripción para la comparación los parques de los distritos de Breña y Pueblo Libre) se diseñó una matriz teniendo como columnas las propiedades visibles de los parques como el ornato, vegetación, acceso, disponibilidad de juegos para niños, seguridad y presencia de basura; y en las filas los nombres de los parques visitados, luego, cada investigador empezó a analizar los valores posibles de cada propiedad visible y expresándolas por un puntaje numérico. Al final se tuvo un promedio. El resultado de lo anterior dicho esta expresado en la Tabla 8.
Para el análisis de distancia, en la Tabla 9 muestra la relación de distancia entre los parques y las entidades educativas en un radio de 100, 200 y $300 \mathrm{~m}$ (solo se toma los parques con más de una entidad educativa relacionada al radio de $100 \mathrm{~m})$.

\section{DISCUSIÓN}

Se pudo apreciar que la relación entre áreas verdes con centros educativos es mínima, lo que lleva a pensar que la importancia que estas tienen en el desarrollo integral de cada estudiante está disminuida o poco comprendida,

Tabla 8. Comparativo de los servicios de los parques de los distritos de Breña y Pueblo Libre.

\begin{tabular}{|c|c|c|c|c|c|c|}
\hline \multirow[b]{2}{*}{ Distrito } & \multicolumn{6}{|c|}{ Servicios } \\
\hline & 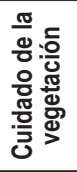 & 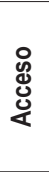 & 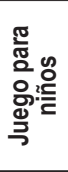 & $\begin{array}{l}\text { 융 } \\
\text { 은 } \\
\text { 엉 }\end{array}$ & 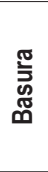 & $\begin{array}{l}\text { 윯 } \\
\text { 동 }\end{array}$ \\
\hline Breña & 3 & 2 & 1 & 1 & 2 & 2 \\
\hline Pueblo Libre & 3 & 2 & 1 & 2 & 2 & 3 \\
\hline
\end{tabular}

Fuente: Elaboración propia.

Tabla 9. Relación de parques con la cantidad de entidades educativas a radios de 100, 200 y $300 \mathrm{~m}$.

\begin{tabular}{|c|c|c|c|c|}
\hline Parque & Distrito & Radio = $100 \mathrm{~m}$ & Radio = $200 \mathrm{~m}$ & Radio $=300 \mathrm{~m}$ \\
\hline Daniel Hernández & Pueblo Libre & 5 & 9 & 12 \\
\hline Bolívar & Pueblo Libre & 4 & 4 & 8 \\
\hline el Carmen & Pueblo Libre & 4 & 7 & 11 \\
\hline Cáceres & Pueblo Libre & 3 & 3 & 4 \\
\hline Francisco Bolognesi (3 de octubre) & Pueblo Libre & 3 & 3 & 9 \\
\hline Isla San Lorenzo & Pueblo Libre & 3 & 3 & 5 \\
\hline Plaza de la Bandera & Breña / Pueblo Libre & 3 & 4 & 8 \\
\hline Murillo & Breña & 3 & 6 & 8 \\
\hline Nathan Rosenbach Heller & Pueblo Libre & 3 & 3 & 3 \\
\hline Pedro Donofrio & Breña & 3 & 5 & 7 \\
\hline Rio Grande & Pueblo Libre & 3 & 7 & 10 \\
\hline Rochdale & Pueblo Libre & 3 & 6 & 8 \\
\hline San Bernardo & Pueblo Libre & 3 & 4 & 7 \\
\hline San Martin & Pueblo Libre & 3 & 9 & 10 \\
\hline Alonso Meza & Pueblo Libre & 2 & 2 & 2 \\
\hline av. Restauración & Breña & 2 & 2 & 6 \\
\hline Colmenares & Pueblo Libre & 2 & 4 & 7 \\
\hline del Rio & Pueblo Libre & 2 & 4 & 4 \\
\hline Enrique León García & Pueblo Libre & 2 & 4 & 5 \\
\hline Galicia & Pueblo Libre & 2 & 2 & 3 \\
\hline Grau & Pueblo Libre & 2 & 3 & 6 \\
\hline Luzuriaga & Breña & 2 & 4 & 6 \\
\hline Maldonado & Pueblo Libre & 2 & 4 & 6 \\
\hline Orellana & Breña & 2 & 5 & 6 \\
\hline Santa María Magdalena & Pueblo Libre & 2 & 6 & 11 \\
\hline Zoila Amoretti & Pueblo Libre & 2 & 2 & 6 \\
\hline
\end{tabular}

Fuente: Elaboración propia. 
así mismo no se ha encontrado información que pueda detallar la cantidad de áreas verdes y condiciones de estas al interior de los centros educativos de ambos distritos. El trabajo de Xiang Xiao et al. (2020) informa sobre la importancia de la proximidad de áreas verdes a colegios ya que está relacionada con la disminución de la presión sanguínea sistólica y la disminución de los valores medio de hipertensión en escolares del noreste de China. Por otra parte Capristán Flores (2017) cita que existe preocupación por el déficit de áreas verdes y sus consecuencias como la exclusión social, pérdida de valor monetario y ecosistémico, y en el caso del distrito de Chaclacayo se busca caracterizar la distribución de estas adecuándose a las necesidades de la población, si bien no cita la cantidad de parques que presenta la zona cita que existe un déficit de $3.6 \mathrm{~m}^{2}$ de áreas verdes por habitante, en Breña se verifica una cantidad menor de parques (21) en comparación con Pueblo Libre (73); para Meza Ortega (2018) puede tratarse de buscar un cambio de actitud desde el nivel de educación primaria, en el distrito de Carabayllo, especialmente con programas sobre concientizar sobre la importancia de las áreas verdes, o también el trabajo en talleres agroecológicos como los desarrollados por Ayón (2017) en el distrito del Agustino en estudiantes de educación secundaria, entonces podemos afirmar que existe relación una preocupación por la presencia de áreas verdes a nivel distrital; para la OMS cada habitante debe contar con al menos $9 \mathrm{~m}^{2}$ de área verde, una propuesta muy alejada de la realidad ya que para Pueblo Libre existe $3.74 \mathrm{~m}^{2} /$ habitante, mientras que para Breña existe $1,01 \mathrm{~m}^{2} /$ habitante.

\section{CONCLUSIONES}

- La cantidad de entidades educativas y el número de alumnado entre los distritos de Breña y Pueblo Libre no es proporcional a sus respectivas áreas: mientras que el distrito de Breña con un área estimada de $3.21 \mathrm{~km}^{2}$ de los cuales $47202 \mathrm{~m}^{2}\left(0.047 \mathrm{~km}^{2}\right)$ son de parques existen 137 entidades educativas que albergan 24144 alumnos, el distrito de Pueblo Libre con un área estimada de $4.64 \mathrm{~km}^{2}$ de los cuales 296 $555 \mathrm{~m}^{2}\left(0.297 \mathrm{~km}^{2}\right)$ son de parques existen 109 entidades educativas albergando 15324 alumnos.

- Del total de área de parques del distrito de Breña, aproximadamente el $70 \%$ de esta área está concentrada entre las avenidas Castrovirreyna, Tingo María, Mariano Cornejo y Aguarico que comprende el $10 \%$ de área de todo el distrito.

\section{REFERENCIAS}

Ayón, E. (2017). Talleres de manejo agroecológico de áreas verdes conciencia ambiental en estudiantes del terreno de secundaria de las instituciones educativas en el distrito de El Agustino Lima. http://repositorio.une.edu.pe/handle/ $\mathrm{UNE} / 1200$

Capristán Flores, R. A. (2017). Manjeo de areas verdes en el distrito de Chaclacayo [Universidad Agraria La Molina. Perú]. In Manejo de áreas verdes en el Distrito de Chaclayo. http://repositorio.lamolina.edu.pe/handle/UNALM/3470
Comisión Nacional de Medio Ambiente (CONAMA). (2002). Áreas verdes en el Gran Santiago. moz-extension:// af9c8ffb-6ba6-4457-980e-9fee00c031c9/enhanced-reader. html?openApp\&pdf=http $\% 3 \mathrm{~A} \% 2 \mathrm{~F} \% 2 \mathrm{Fmetadatos}$.mma. gob.cl $\% 2$ Fsinia $\% 2$ FF $1350 . p d f$

Compañía peruana de estudios de mercados y opinión pública S.A.C. (2019). Perú 2019: Población según segmentos de edad. moz-extension://af9c8ffb6ba6-4457-980e-9fee00c031c9/enhanced-reader. html?openApp\&pdf=http\%3A\%2F\%2Fcpi.pe \% 2Fimages $\% 2$ Fupload\%2Fpaginaweb\%2Farchivo\%2F $26 \% 2 \mathrm{Fmr}$ poblacional_peru_201905.pdf

ESCALE. (2012). Estadística de localidad Educativa Ministerio de Educación del Perú. Plano de Instituciones Educativas Básica Regular y Alternativa UGEL - 03 Lima. http:// escale.minedu.gob.pe/carta-educativa/-/document library display/z0Kj/view/967851/7919?_110_INSTANCE_ $\mathrm{z} 0 \mathrm{Kj}$ _redirect $=\mathrm{http} \% 3 \mathrm{~A} \% 2 \mathrm{~F} \% 2 \mathrm{Fescale}$. minedu.gob. pe $\% 2$ Fcarta-educativa $\% 2$ F- $\% 2$ Fdocument_library_ display\%2Fz0Kj\%2Fview\%2F967851

ESCALE. (2019). Mapa de Escuelas Ministerio de Educación del Perú. Estadística de Localidad Educativa. http://sigmed. minedu.gob.pe/mapaeducativo/

Flores-Xolocotzi, R., \& González-Guillén, M. D. J. (2019). Planificación de sistemas de áreas verdes y parques públicos. Revista Mexicana de Ciencias Forestales, 1(1), 17-24. https://doi.org/10.29298/rmcf.v1i1.649

Herrera, R., Markevych, I., Berger, U., Genuneit, J., Gerlich, J., Nowak, D., Schlotz, W., Vogelberg, C., Von Mutius, E., Weinmayr, G., Windstetter, D., Weigl, M., Heinrich, J., \& Radon, K. (2018). Greenness and job-related chronic stress in young adults: A prospective cohort study in Germany. BMJ Open, 8(6). https://doi.org/10.1136/bmjopen-2018-021599

Jia, X., Yu, Y., Xia, W., Masri, S., Sami, M., Hu, Z., Yu, Z., \& Wu, J. (2018). Cardiovascular diseases in middle aged and older adults in China: the joint effects and mediation of different types of physical exercise and neighborhood greenness and walkability. Environmental Research, 167, 175-183. https:// doi.org/10.1016/j.envres.2018.07.003

Lamela, A., M. F. y S. M. (2011). En búsqueda de unas recomendaciones urbanisticas mundiales de densidad $y$ espacios verdes. Nimbus, (27-28). https://www. researchgate.net/publication/228512073_En_busqueda de_unas_recomendaciones_urbanisticas_mundiales_de densidad_y_espacios_verdes

LIMA CÓMO VAMOS. (2014). QUINTO INFORME DE PERCEPCIÓN SOBRE CALIDAD DE VIDA ENCUESTA FICHA TÉCNICA.

Markevych, I., Thiering, E., Fuertes, E., Sugiri, D., Berdel, D., Koletzko, S., Von Berg, A., Bauer, C. P., \& Heinrich, J. (2014). A cross-sectional analysis of the effects of residential greenness on blood pressure in 10-year old children: Results from the GINIplus and LISAplus studies. BMC Public Health. https://doi.org/10.1186/1471-2458-14-477

Meza Ortega, D. (2018). Implementación de una campaña social de concientización sobre las áreas verdes en los alumnos de sexto grado del nivel primario turno tarde de la I.E $\mathrm{N}^{\circ}$ 2037 Ciro Alegria del distrito de Carabayllo, en el año 2018. Universidad César Vallejo. http://repositorio.ucv.edu.pe/ handle/20.500.12692/34221 
Nieuwenhuijsen, M.J., Khreis, H,, Triguero-Mas, M., Gascón, M., Dadvand, P. (2017). Fifty Shades of Green: Pathway to Healthy Urban Living. Epidemiology 28. https://www. semanticscholar.org/paper/Fifty-Shades-of-Green\%3APathway-to-Healthy-Urban-Nieuwenhuijsen-Khreis/ f23a3b48a2f54482fbabdbe10b08a8671a0ad164

Pérez, E. (2011). Paisaje Urbano en Nuestras Ciudades. Bitácora Urbano-Territorial. https://revistas.unal.edu.co/index.php/ bitacora/article/view/18810

Sander, H. A., Ghosh, D., \& Hodson, C. B. (2017). Varying agegender associations between body mass index and urban greenspace. Urban Forestry and Urban Greening, 26, 1-10. https://doi.org/10.1016/j.ufug.2017.05.016

Sistema Nacional de Información Ambiental-SINIA. (2019). Indicador: Superficie de área verde urbana por habitante en Lima Metropolitana. Ministerio Del Ambiente Del Perú -MINAM. https://sinia.minam.gob.pe/indicador/998

Sorensen, M., Barzetti, V., Keipi, K., \& Williams, J. R. (1998). Manejo de las areas verdes urbanas | Publications. https:// publications.iadb.org/es/publicacion/15940/manejo-de-lasareas-verdes-urbanas

Van Aart, C. J. C., Michels, N., Sioen, I., De Decker, A., Bijnens, E. M., Janssen, B. G., De Henauw, S., \& Nawrot, T. S. (2018). Residential landscape as a predictor of psychosocial stress in the life course from childhood to adolescence. Environment International, 120, 456-463. https://doi. org/10.1016/j.envint.2018.08.028
Vera, L., Ramírez, M. y Pértile, V. (2013). Disponibilidad de espacios verdes en la ciudad de Resistencia: Estudiomediante la aplicación de Sistemas de Información Geográfica (SIG). VII Seminario de Políticas Urbanas, Gestión Territorial y Ambiental Para El Desarrollo Local de Instituto de Planeamiento Urbano y Regional (IPUR) Brian Alejandro Thomson (BAT) FAU - UNNE, Resistencia, Argentina. https://doi.org/10.1017/CBO9781107415324.004

Wolch, J. R., Byrne, J., y Newell, J. P. (2014). Urban green space, public health, and environmental justice: The challenge of making cities 'just green enough.' Landscape and Urban Planning, 125, 234-244. https://escholarship.org/uc/ item $/ 8 \mathrm{pf} 8 \mathrm{~s} 47 \mathrm{q}$

Xiang Xiao, Yang, B. Y., Hu, L. W., Markevych, I., Bloom, M. S., Dharmage, S. C., Jalaludin, B., Knibbs, L. D., Heinrich, J., Morawska, L., Lin, S., Roponen, M., Guo, Y., Lam Yim, S. H., Leskinen, A., Komppula, M., Jalava, P., Yu, H. Y., Zeeshan, M., ... Dong, G. H. (2020). Greenness around schools associated with lower risk of hypertension among children: Findings from the Seven Northeastern Cities Study in China. Environmental Pollution, 256. https://doi. org/10.1016/j.envpol.2019.113422 